\title{
Improving the Connectivity of Heterogeneous Multi-Hop Wireless Networks
}

\author{
Tae-Hoon Kim, David Tipper, and Prashant Krishnamurthy \\ Graduate Networking and Telecommunications Program \\ University of Pittsburgh, Pittsburgh PA, USA \\ Email: tak11@pitt.edu, \{dtipper, prashant\}@sis.pitt.edu
}

\begin{abstract}
Heterogeneous conditions can occur in multi-hop wireless networks due to a variety of factors such as variations in transmission power and signal propagation environments. Directed links can occur when the environment and/or the nodes are heterogeneous. In this paper, we examine the network connectivity for heterogeneous multi-hop wireless networks and propose an algorithm to identify the connectivity of the network. We follow this with a numerical study of the connectivity in random topologies. Lastly, we propose two schemes for constructing additional links to enhance the connectivity of the network. Our proposed schemes identify the links to be improved or created via a cluster based approach.
\end{abstract}

Index Terms-Connectivity, heterogeneous multi-hop wireless networks

\section{INTRODUCTION}

Multi-hop wireless networks are expected to become an important part of the communications landscape. Examples include wireless mesh networks (WMNs) [1], vehicular ad-hoc networks (VANETs) [2], and mobile ad-hoc networks (MANETs) [3]. In multi-hop wireless networks, the nodes must cooperate to dynamically establish routes using wireless links and routes may involve multiple hops with each node acting as a router. In many multi-hop network scenarios the nodes can move arbitrarily or the interference can change dynamically resulting in topology changes. Multi-hop wireless networks also inherit the traditional problems of wireless communications, which when combined with mobility and lack of infrastructure makes their design and development challenging [1-3]. A basic problem in many multi-hop wireless networks is maintaining connectivity. A network is connected if all nodes have a communication path to each other. Similarly, a network is k-connected if there are k-disjoint paths between every pair of nodes. Maintaining connectivity is difficult due to the unstructured nature of the topology and the occurrence of link and node failures due to interference, mobility, radio channel effects and battery life [1-3].

Many researchers have studied multi-hop wireless network connectivity determining conditions under which connectivity $[3,8]$ and k-connectivity [9-11] can be inferred probabilistically or assured asymptotically. The focus has largely been on what combination of node density and power range are required to provide $k$-node connectivity in a specific deployment scenario for a homogenous network. A major weakness of this work is the assumption of a homogeneous

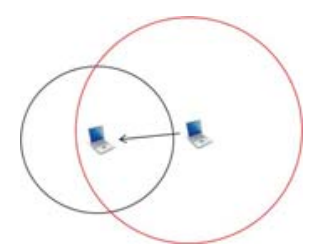

(a) Different Tx power levels

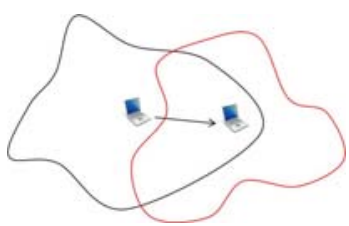

(b) Non-uniform Tx range
Fig. 1. Directional links in heterogeneous wireless networks

network context where nodes have identical properties and inhabit a uniform environment (e.g., identical transmission power, battery life, radio propagation ranges, antennas, etc.). Measurement studies [12] have shown that many of the assumptions in the homogeneous context are inaccurate. In particular it was noted that real networks can have directional links. For example, if each node has different transmission power, then their transmission range differs and a directional link can result as shown in Figure 1(a). Similarly, in Figure 1(b) a directional link results from nodes having non-identical signal propagation due to differences in the local environment (e.g., trees, buildings, etc.).

In this paper, we look at how one can develop techniques to improve the connectivity of sparse heterogeneous multi-hop wireless networks without increasing the node density. Our approach is to determine the topological connectivity critical points that need to be improved to attain/maintain at least 1-connectivity. We propose a novel algorithm for determining the connectivity based on results from algebraic graph theory. Using the critical points identified by our connectivity algorithm, we propose two techniques to improve the connectivity of the network by adjusting the transmission power of nodes around a critical point in order to create additional links in the network.

The rest of the paper is organized as follows. In Section II, we propose an algorithm to determine the connectivity of heterogeneous networks. Numerical results utilizing the proposed algorithm to study the effects of network density on the connectivity are given. Then, we propose two network link modification schemes in Section III to improve the network connectivity without changing the node density. Simulation results illustrating the effectiveness and tradeoffs of the proposed connectivity improvement schemes are also given. Lastly, we present our conclusions in Section IV. 


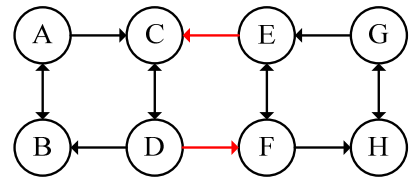

(a) 1-Connected

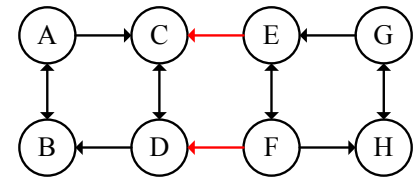

(b) Disconnected
Fig. 2. Sample 8 node heterogeneous network topologies

\section{Heterogeneous NeTwork CONNECTIVITY}

A distinguishing characteristic of homogeneous networks is that the set of multi-hop paths between a pair of nodes are the same in each direction, while this may not be the case in a heterogeneous network. For example, in the network of Figure 2 (a), the path from node $\mathrm{A}$ to $\mathrm{H}$ is $\mathrm{A} \rightarrow \mathrm{C} \rightarrow \mathrm{D} \rightarrow \mathrm{F} \rightarrow \mathrm{H}$ whereas the path from $\mathrm{H}$ to $\mathrm{A}$ is $\mathrm{H} \rightarrow \mathrm{G} \rightarrow \mathrm{E} \rightarrow \mathrm{C} \rightarrow \mathrm{D} \rightarrow \mathrm{B} \rightarrow \mathrm{A}$ (i.e., $\left.P A T H_{A \rightarrow H} \neq P A T H_{H \rightarrow A}\right)$. Another effect of directed links in the topology is that communications may be one way. For example in the network of Figure 2 (b), nodes A, B, C, and D can receive data from $\mathrm{E}, \mathrm{F}, \mathrm{G}$, and $\mathrm{H}$ through the links $\mathrm{E} \rightarrow \mathrm{C}$ and $\mathrm{F}$ $\rightarrow \mathrm{D}$. However, the opposite direction is not available such that A, B, C, or D, can communicate with E, F, G, or H. Given that directional links can occur in heterogeneous networks we define the connectivity between a pair of nodes as requiring that they be bi-communicable. Specifically we have the following. Definition 1. Bi-communicable: $A$ pair of nodes is bi-communicable if and only if both nodes can receive information from each other. Note that bi-communicable doesn't require that paths between the nodes in question have the same set of intermediate nodes.

This definition indicates that node $i$ and $j$ are connected if and only if node $i$ can receive data sent by $j$ and vice versa. Based on this connectivity definition, we introduce a modified definition of a link between two nodes in a network.

Definition 2. Valid Link: A pair of nodes $i$ and $j$ have a valid link if and only if a direct link exists in both directions or at least one direct link in either direction exists and at least one multi-hop path in the other direction is available.

Based on Definitions 1 and 2 we define a partition of the network as follows.

Definition 3. Partitioned Network: A network is considered partitioned if one or more nodes do not have bi-directional connectivity to the rest of the nodes in the network.

Hence a network is 1-connected if and only if all pairs of nodes have at least one path between them in both directions. Here we propose an algorithm for a network node to test the overall connectivity of the topology when directed links exist in the network.

\section{A. Connectivity Test}

Consider an arbitrary multi-hop wireless network of $N$ nodes. Let $G=(V, E)$, be the graph of the topology where $V$ is the set of nodes and $E$ is the set of links. The graph $G$ can be represented by the $N \times N$ adjacency matrix $A(t)$ at time $t$,

$$
A(t)=\left[\begin{array}{cccc}
a_{11}(t) & a_{12}(t) & \cdots & a_{1 N}(t) \\
a_{21}(t) & a_{22}(t) & \cdots & a_{2 N}(t) \\
\vdots & \vdots & & \vdots \\
a_{N 1}(t) & a_{N 1}(t) & \cdots & a_{N N}(t)
\end{array}\right]
$$

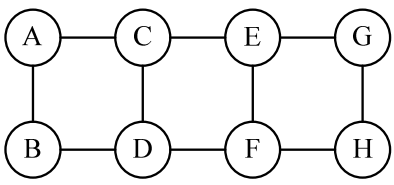

(a) Adjusted 1-Connected
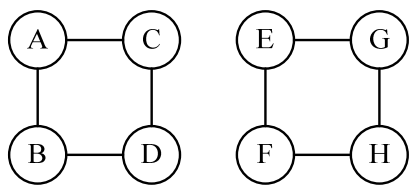

(b) Adjusted Disconnected
Fig. 3. Adjusted network topologies corresponding to Fig. 2

where

$$
a_{i j}(t)= \begin{cases}1, & \text { if link } i \text { to } j \text { exist } \\ 0, & \text { otherwise }\end{cases}
$$

The link connectivity $a_{i j}(t)$ between two nodes depends on their radio range and can be determined by nodes locally through the exchange of "Hello" or probing packets. For now we assume a homogeneous network scenario where all links are bidirectional (i.e., $a_{i j}(t)=1 \rightarrow a_{j i}(t)=1$ ). Thus, $A(t)$ is a symmetric matrix. Let $d_{i}(t)$ denote the degree of node $i \in N$ at time $t$ (i.e., $d_{i}(t)$ equals the number of neighbor nodes of $i$ ). We define $D$ as the diagonal matrix consisting of the degree of each node (i.e., $\left.D(t)=\operatorname{diag}\left(d_{i}(t)\right)\right)$. The Laplacian matrix $L(t)$ of a graph is defined in terms of the adjacency matrix $A(t)$ and nodal degree matrix $D(t)$ as

$$
L(t)=D(t)-A(t)
$$

The eigenvalues $\lambda=\left[\lambda_{1}, \lambda_{2}, \ldots \lambda_{N}\right]$ of $L(t)$ form the Laplacian spectrum of the graph. The eigenvalues $\lambda$ and their associated eigenvectors $\mathbf{X}=\left[\mathrm{x}_{1}, \mathrm{x}_{2}, \ldots \mathrm{x}_{\mathrm{N}}\right]$ can be computed from $(\mathrm{L}-\lambda \mathbf{I}) \mathbf{X}=0$ using a number of well known efficient numerical methods. In algebraic graph theory [13] it has been shown that zero is always an eigenvalue of $L$ and the number of zero eigenvalues is equal to the number of connected components of the network $N_{C L}$. Thus the network is connected (i.e., at least 1-connectivity) if the Laplacian matrix of the topology has a single zero eigenvalue (i.e., if second smallest eigenvalue is positive $\rightarrow N_{C L}=1$ ). Here we apply this result in constructing a test for connectivity in heterogeneous multi-hop wireless networks. The adjacent matrix can be obtained by use of the topology information periodically gathering by proactive routing protocol or reactive routing protocol that exchange local connectivity periodically. The possible existence of directed links in the topology means that one can not directly apply the result. In order to force the adjacency matrix $A(t)$ to have a symmetric form we apply Definition 2 above in determining the link connectivity. Specifically, we require all links to be valid links and modify the link connectivity in a logically adjusted topology adjacency matrix $A$ to reflect this. For example, the sample topologies of Figure 2 after adjustment will have the corresponding logical topologies shown in Figure 3. Note, that after adjustment of the network topology the resulting $A(t)$ is symmetric and one can apply a test on the Laplacian eigenvalues to determine the network connectivity. The connectivity test procedure is given in algorithmic form below.

\section{Heterogeneous Connectivity Test Algorithm (h-CTA)}

Step 1: Identify any directed links in the topology from the adjacency matrix $A(t)$. If none, set $A^{\prime}(t)=A(t)$ and go to step 3.

Step 2: Form adjusted adjacency matrix $A^{\prime}(t)$ containing only valid links. Specifically, for each link $i-j$ such that $a_{i j} \neq a_{j i}$, 


$$
\begin{aligned}
& a_{i j} \in A \\
& \text { if } \begin{cases}a_{i j}=1 \wedge \operatorname{Path}(j \rightarrow i) \text { exists, } & a_{\imath \jmath}{ }^{\prime}=a_{\jmath \imath}{ }^{\prime}=1 \\
a_{j i}=1 \wedge \operatorname{Path}(i \rightarrow j) \text { exists, }, & a_{\imath \jmath}=a_{\jmath \imath}{ }^{\prime}=1 \\
\text { Otherwise, } & a_{\imath \jmath}=a_{\jmath \iota}{ }^{\prime}=0\end{cases}
\end{aligned}
$$

where $A$ is the adjusted adjacent matrix.

Step 3: Compute the eigenvalues of the Laplacian matrix $L=$ $D-A$, where $D$ is the degree matrix corresponding to $A$

Step 4: Determine the number of zero eigenvalues among the Laplacian spectrum. If $N_{C L}=1$ then the network is connected, otherwise it is partitioned into $N_{C L}$ components networks.

For a network of $N$ nodes and $E$ links of which $K$ are directed, it can be shown that the time complexity of $h-C T A$ is $O(K(E+N$ $\log N)+N^{2}$ ). The algorithm can be implemented at any network node having adjacency matrix information However, if the network is partitioned according to Definition 3, all nodes cannot exchange connectivity information. For example, in the network of Figure 2 (b) cluster 2 (i.e., $C L_{2}=\{\mathrm{E}, \mathrm{F}, \mathrm{G}, \mathrm{H}\}$ ) cannot receive connectivity information of cluster 1 (i.e., $C L_{1}=$ $\{\mathrm{A}, \mathrm{B}, \mathrm{C}, \mathrm{D}\})$ while cluster 1 can receive and obtain the connectivity information of cluster 2 . Since the nodes in cluster 1 can obtain the global adjacency information, any node in cluster 1 can execute $h$-CTA to determine the overall connectivity. In Section III we show a node with in a cluster such as 1 in Figure 2(b) can find which link should be improved so that the cluster 2 can receive the connectivity information of cluster 1 . If there exists other weakly connected clusters, the cluster that can receive the most information can do initiate the h-CTA procedure to determine which links need to be added to provide connectivity. A network such as Figure 2(b) is termed weakly connected and this is our focus in the paper. Once $A(t)$ is found, $k$-connectivity may be also examined by the algebraic connectivity. However, it is out of scope in this paper.

\section{B. Numerical Study}

We utilized the $h$-CTA algorithm to study the connectivity of heterogeneous multi-hop wireless network topologies. Here we discuss typical results for the case of random topologies. Using the ns2 simulator, we generated random topologies with different number of nodes (i.e., 50, 75, and 100) in a network area of $1500 \times 1500 \mathrm{~m}^{2}$. The nodes were independently distributed according to a uniform [0-1500] random variable in the network area. We adopted baseline parameters from $802.11 \mathrm{~b}$ equipment (i.e., average transmit power $P_{T}=15 \mathrm{dBm}$, receiver sensitivity threshold $P_{R S S T}=-90 \mathrm{dBm}$ ). The basic transmission range was determined using a simple path loss model

$$
P_{R}(\mathrm{dBm})=P_{T}(\mathrm{dBm})-10 \alpha \log (d)
$$

where $\alpha$ is the path loss exponent and $d$ is the distance between a pair of nodes in meters. A path loss exponent of $\alpha=4.3788$ was used, which results in a circular coverage area with radius 250 meters. A heterogeneous network was created by varying either the transmission power or propagation model for each node or both factors. Each node $i$ selects its transmission power $P_{T i}$ according to a uniform $[13.5 \mathrm{dBm}-16.5 \mathrm{dBm}]$ random variable. The resulting maximum transmission range $R$ is uniform between $231 \mathrm{~m}$ and $270.5 \mathrm{~m}$. Non-uniform signal propagation between nodes was modeled using the Quasi-Unit
Disk Graph (Q-UDG) model. In the Quasi-UDG model, a link exists between two nodes if the inter-nodal distance $d$ is less than $\beta R$, where $R$ is the maximum transmission range of the node and $\beta$ is the Q-UDG factor $(0 \leq \beta \leq 1)$. For distances $d$ greater than $R$, there is no connectivity. However, for $\beta R \leq d \leq$ $R$, the link will exist with probability $(R-d) /(R-\beta R)$. We selected different $\beta$ parameter values of $0.5,0.75,0.85$ and 1.0 to show how irregular propagation affects the connectivity. Figure 4 shows $95 \%$ confidence intervals on the $P$ (connected network) determined with h-CTA versus the node density for two cases of node power assignment: (a) homogenous with $P_{T i}$ $=15 \mathrm{dBm}$ at each node $i$ and (b) heterogeneous with $P_{T i}$ drawn from a uniform $[13.5 \mathrm{dBm}-16.5 \mathrm{dBm}]$ random variable for each node. Each point in Figure 4 is determined from 4000 independent simulation runs. From Figure 4(a), notice that for the case of a homogeneous network (i.e., fixed $P_{T}=15 \mathrm{dBm}, \beta=$ $1.0)$ the network is connected (i.e., $P($ connected network $)=1$ ) for all node densities considered. In contrast, the $P$ (connected network) is almost zero for all network densities for both homogeneous (4(a)) and heterogeneous power assignments (4(b)) when $\beta=0.5$. This is because the average transmission range is only 187.5 meters. However, the network connectivity increases as $\beta$ increases. For example, in Figure 4(a) the probability of connectivity for a 100 node network is estimated from the simulation as 0.0043 with $\beta=0.5,0.226$ with $\beta=0.75$,

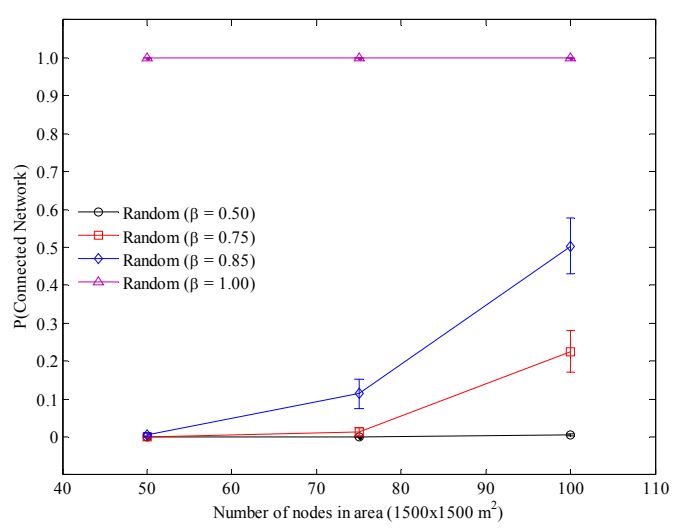

(a) Random networks with homogeneous transmission power

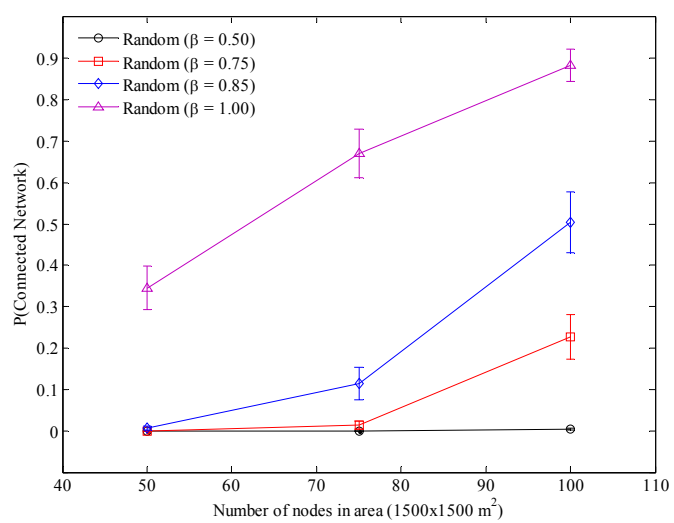

(b) Random networks with heterogeneous transmission power

Fig. 4. Probability of connectivity in random network topologies 
and 0.504 with $\beta=0.85$. Similarly for a fixed $\beta$ the network connectivity increases with the node density, for example in Figure 4 (a) with $\beta=0.85$, the probability of connectivity is 0.0063 at 50 nodes, 0.1145 at 75 nodes, and 0.504 at 100 nodes. In comparing homogeneous power assignment with heterogeneous assignment (i.e., 4(a) vs. 4(b)) we see that only for the $\beta=1$ case does the power assignment result in a significant difference in the connectivity. For the other values of $\beta$, the Q-UDG propagation effect dominates and the power assignment has little effect on the results (i.e., the confidence intervals on the results overlap). From Figure 4 one can clearly see that the connectivity in heterogeneous network is considerably lower than the homogenous case no matter what the cause of the heterogeneity.

\section{CONNECTIVITY IMPROVEMENT SCHEMES}

We propose schemes that for an unconnected heterogeneous network identify the links whose addition will connect the network. The approach is based on identifying the isolated groups (i.e. clusters) of nodes and determining the links required to connect between clusters. Consider a network partitioned into $N_{C L}$ weakly connected clusters (i.e., each cluster has at least a directed link to some other cluster). The Laplacian matrix $L$ with re-labeled node IDs can be written as

$$
L=\left(\begin{array}{ccc}
L_{1} & \cdots & 0 \\
\vdots & \ddots & \vdots \\
0 & \cdots & L_{N C L}
\end{array}\right)
$$

where each $L_{k}$ represents the Laplacian matrix of a connected cluster $C_{k}$ of nodes. There are $N_{C L}$ zeros in the eigenvalue set $\lambda$ $=\left[\lambda_{1}, \lambda_{2}, \ldots \lambda_{\mathrm{N}}\right]$ of $L(t)$. From the $N_{C L}$ eigenvectors $x_{i}$ associated with the zero eigenvalues one can determine the nodes in each cluster. Specifically the nodes in a cluster will have non-zero values in the eigenvector associated with a zero eigenvalue [15] Once the sets of member nodes in each isolated cluster are identified, a cluster adjacency matrix, $C L_{-} A$, can be determined which represents how the clusters are asymmetrically connected. The cluster adjacency matrix can be determined by:

$$
C L_{-} a_{i j}=\left\{\begin{array}{cc}
1, & \forall k \in C L_{i} \wedge \forall l \in C L_{j}, \exists A_{k l}=1 \\
0, & \text { otherwise }
\end{array}\right.
$$

where $C L_{i}$ is a nodes set of each cluster $i$, and $A$ is the network adjacency matrix. The cluster adjacency matrix provides information about which cluster needs to be connected to another cluster to provide network connectivity. We propose two schemes to identify the links needed to reconnect the network based on given cost constraints: (1) Simple Merging Scheme (SMS) and (2) Cost Optimized Merging Scheme (COMS). The link cost is the cost to improve the link so that bi-communication can occur. Note that a variety of techniques such as node movement, transmission power, directional antenna, and etcetera can be used to add a link to the network. In this paper, we manipulate the transmission power to improve the links in question and the link cost constraint $L C_{\text {limit }}$ in terms of distance is determined from the maximum transmission power together with the propagation model.

\section{A. Simple Merging Scheme (SMS)}

The Simple Merging Scheme (SMS) tries to add links until each isolated cluster has a bi-directional connection with it's neighbor clusters. The algorithm finds the set of local minimum cost links between an isolated cluster and a neighbor cluster using distance information. Specifically between each pair of neighbor clusters SMS finds the minimum distance links that are within the maximum possible transmission range as determined by $L C_{\text {limit }}$. The distance between nodes in isolated clusters can be obtained by Global Positioning System (GPS) or localization techniques $[14,15]$. SMS can be implemented locally by the cluster heads (nodes with a directed link to another cluster) in each cluster. SMS can be put in algorithm form as below.

\section{Simple Merging Scheme (SMS)}

Step 1 : Perform $h$-CTA to determine if the network is partitioned, if Yes go to step 2, otherwise STOP.

Step 2: From the h-CTA results determine the number of clusters $N_{C L}$ and the node sets of each cluster, $C_{i}$, and the cluster adjacency matrix $C L A$.

Step 3: Find the set of minimum cost links between each pair of isolated clusters, $M L C_{i j}=\operatorname{Min}\left\{L C(k, l) ; k \in C_{i}, l \in C_{j}\right\}$, where $M L C_{i j}$ is minimum cost link from cluster $i$ to $j$ and $L C(k, l)$ is the link cost between node $k$ in cluster $i$ and $l$ from cluster $j$. Exclude the existing links.

Step 4 : Select the adequate links based on constraints such that the link direction satisfying $C L \_a_{i j}=0$ and $M L C_{i j}<L C_{\text {limit }}$.

\section{B. Cost Optimized Merging Scheme (COMS)}

In order to provide a benchmark comparison to SMS we developed the Cost Optimized Merging Scheme (COMS) which finds the set of globally minimum cost links between isolated clusters, $M L C=\left\{\right.$ for all $i$ and $\left.j ; M L C_{i j}\right\}$ while satisfying the cost constraint $L C_{\text {limit }}$. This process is very time consuming since it has to check every combination of $2^{N_{C L}}-1$ links and its computation time is exponential. Therefore, before solving the optimization problem we add a step to merge small clusters with one node to the nearest larger cluster. COMS can be implemented in a centralized fashion at a super node and put in algorithm form as follows.

\section{Cost Optimized Merging Scheme (COMS)}

Step 1 : Perform $h$-CTA to determine if the network is partitioned, if Yes go to step 2, otherwise STOP.

Step 2 : From the h-CTA results determine the number of clusters $N_{C L}$ and the node sets of each cluster, $C_{i}$, and the cluster adjacency matrix $C L A$.

Step 3 Merge the clusters with a single member to the nearest bigger cluster. Add these links to an improvement required set of links. If this process resolves the partition problem, STOP. Otherwise, go on step 4.

Step 4 Find the minimum Total Cost (TC) Min: $\sum_{i=1}^{l} L C_{l_{i s} l_{i d}}$, set of links to provide bi-communicable connectivity between to all nodes.

Step 5: Add the set of links from step 4 into the set of links from step 2.

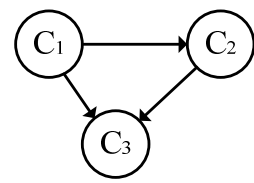

(a) Original clusters

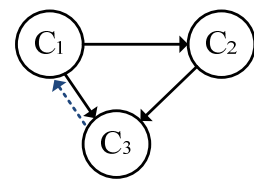

(b) COMS

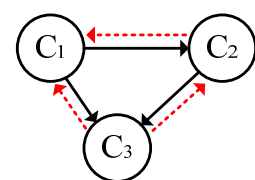

(c) SMS
Fig. 5. Added links by SMS (RED) and COMS (BLUE) 


\section{Comparison of Cluster Merging Schemes}

Figure 5 shows a simple example illustrating the differences between SMS and COMS. Figure 5 (a) shows the original connections between the three clusters of a network. The nodes in cluster $C_{3}$ can obtain topology information from $C_{1}$ and $C_{2}$ and one can initiate COMS. Figure 5(b) shows the topological results of running the COMS algorithm. Observe that only a single directed link is added to provide bi-communicable connectivity to all clusters. In contrast the topology after running SMS is shown in Figure 5(c). Obviously SMS adds more links then COMS and thus costs more but it has the advantages of being distributed and making the network more robust to failures. Note that one node in $C_{2}$ and one in $C_{3}$ will independently initiate the SMS algorithm.

As a more extensive, evaluation and comparison of SMS and COMS, we conducted a set of simulation based experiments similar to those reported in Section II B. Specifically, using the ns2 simulator we generated random topologies with different node densities (i.e., 50, 75, and 100) in a network area of 1500

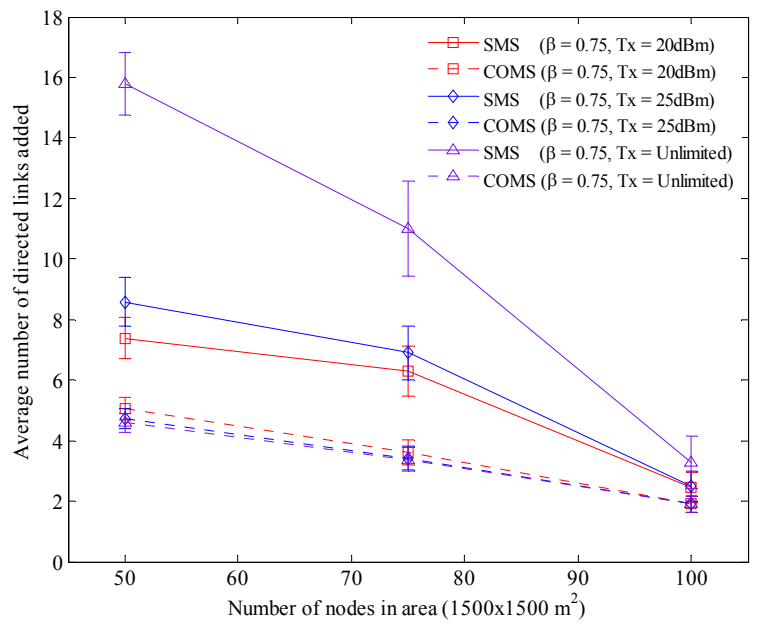

(a) Average number of directed links added for $\beta=0.75$

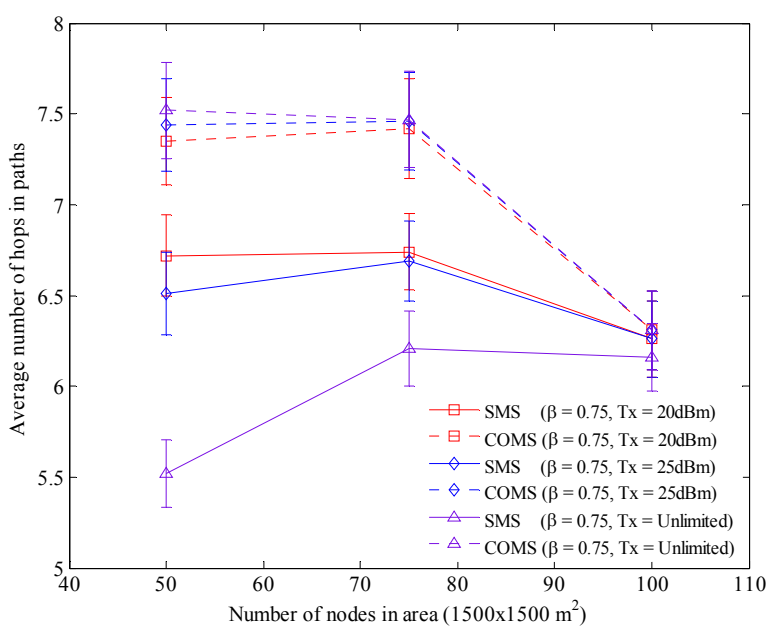

(c) Average number of hops in paths for $\beta=0.75$ $\times 1500 \mathrm{~m}^{2}$. The nodes were independently distributed according to a uniform [0-1500] random variable in the area. We adopted baseline parameters from $802.11 \mathrm{~b}$ equipment (e.g., $P_{R S S T}=-90$ $\mathrm{dBm})$. Heterogeneous conditions were created by having each node $i$ selects its transmission power $P_{T i}$ according to a uniform $[13.5 \mathrm{dBm}-16.5 \mathrm{dBm}]$ random variable and created non-uniform signal propagation between nodes using the Quasi-Unit Disk Graph (Q-UDG) model with $\alpha=4.3788, \beta=$ 0.75 or $\beta=0.85$. We randomly generated topologies in this fashion until 50 weakly connected topologies were found for each node density. For each weakly connected topology both SMS and COMS were implemented to improve the connectivity. We examined three maximum node transmission powers constraints namely: $20 \mathrm{dBm}, 25 \mathrm{dBm}$, and unlimited. In the unlimited power case for COMS the power was increased to the minimum power required to provide the minimum cost links necessary for bi-communicable 1-connectivity. In the unlimited power case for SMS, the power was increased until

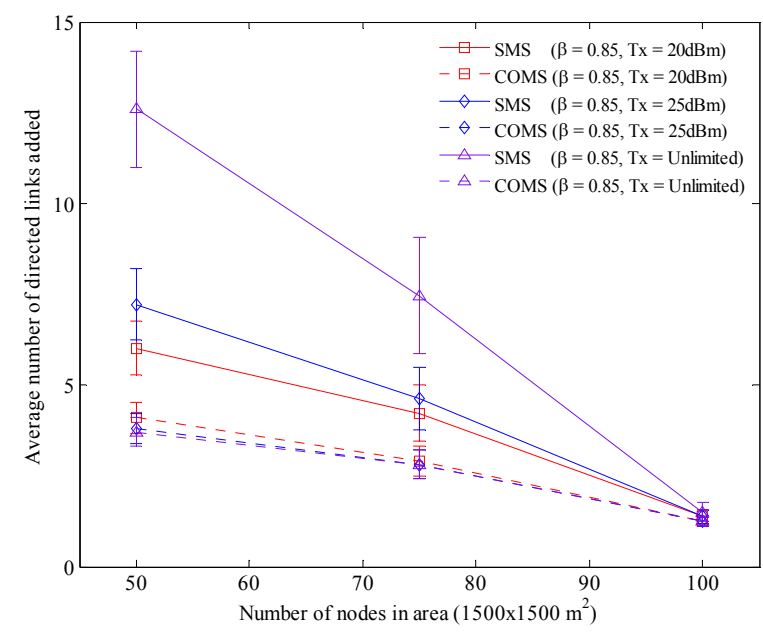

(b) Average number of directed links added for $\beta=0.85$

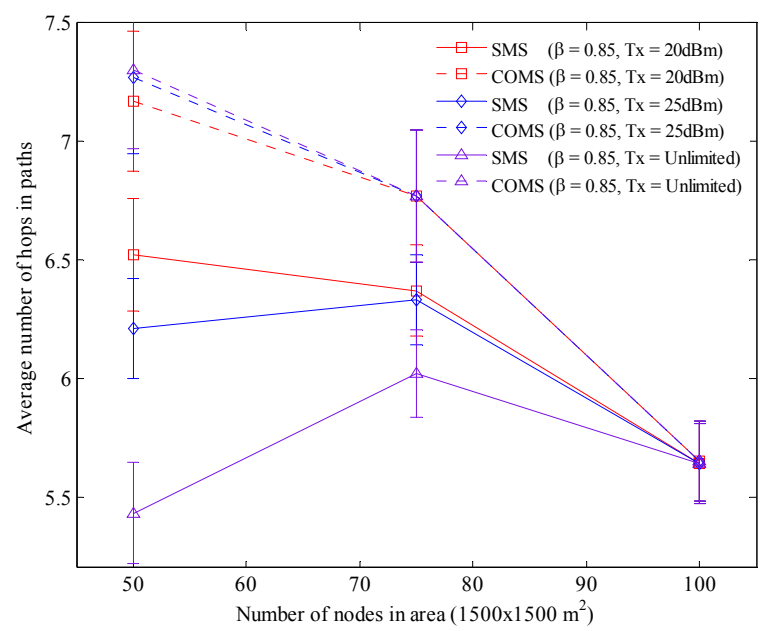

(d) Average number of hops in paths for $\beta=0.85$

Fig. 6. Comparison of SMS and COMS in average number of directed adding links and hops in paths with $95 \%$ of confidence interval 
the minimum power necessary to add links that result in a full mesh of links between all clusters. While the unlimited transmit power case is impractical it provides a benchmark scenario for the results.

Figure 6 shows typical simulation results. For the three maximum transmit power limit investigates after applying SMS or COMS all topologies were at least bi-communicable 1-connected. Figure 6 (a) and (b) show the average number of directed links added to the network versus network density for both SMS and COMS. As expected, when the network density increases fewer links are required to be added in order to provide connectivity regardless of whether SMS or COMS is used. For example, COMS adds an average of 5.52 links in 50 node networks and 1.96 links in 100 node networks with $\beta=$ 0.75 and a maximum transmit power limit of $20 \mathrm{dBm}$. Comparing SMS and COMS one can see that COMS needs fewer links to provide connectivity. In examining the effects of the maximum transmit power limit one can see that the two schemes behave differently. For SMS as the power increases more links are added beyond the minimum needed for 1-connectivity. In the extreme case of unlimited available transmit power, links are added until the clusters are interconnected with a full mesh of links. In contrast, COMS always chooses the cost optimized minimum number of additional links. Hence, the mean number of additional links decreases slightly with increasing maximum power limit. Further comparing the effect of $\beta$ on the results one can see that as $\beta$ decreases and the signal propagation becomes more irregular the average number of directed links need to provide connectivity increases.

Figure 6(c) and (d) show how the average hop count in source-destination paths changes versus the network density for both schemes. The average hop count of all paths between every pair of nodes is considered. From the figures SMS outperforms COMS for all network densities, $\beta$ values, and restoration restrictions. For example, with a $20 \mathrm{dBm}$ maximum power level restriction, the average hop count for SMS is 6.72 and COMS is 7.35 for a 50 node network with $\beta=0.75$. Since COMS finds the minimum number of additional links to provide connectivity, while SMS generate as many as it could, the average hop count by SMS should be always smaller than COMS. On the other hand, in terms of the average link cost COMS always results in a lower average cost and the difference is more significant as the maximum power level limit increases.

Note that, the worse case maximum number of directed links to connect $N_{C L}$ clusters is $2\left(N_{C L}-1\right)$. The time complexity of SMS can be shown to be $O\left(N_{C L}^{2}\right)$ and that of COMS is $O\left(k \times N_{C L}^{2}\right)$ where $k$ is a number of possible link combinations. The range of the number of link combinations is 1 to $2\left(N_{C L}-1\right)$ and $k$ can be computed by

$$
k=\sum_{l=1}^{2\left(N_{C L}-1\right)}\left(\begin{array}{c}
\operatorname{lm} x \\
l
\end{array}\right), \quad \text { where, } \operatorname{lm} x=2\left(\begin{array}{c}
N_{C L} \\
2
\end{array}\right)
$$

Therefore, the computation time of SMS is smaller than that of COMS. If the number of isolated clusters $\mathrm{N}_{\mathrm{CL}}$ increases, the computation time of COMS increases more quickly than SMS.

\section{CONCLUSIONS}

Many 1-connected homogeneous wireless networks become disconnected when the network becomes heterogeneous. In this paper we have proposed a new algorithm to determine the connectivity of heterogeneous wireless networks. The results of a simulation based numerical study utilizing our proposed algorithm to examine the effects of several factors (variations in power levels, irregular signal propagation, and network nodal density) on connectivity are presented. Further we propose two connectivity management schemes SMS and COMS to add additional links to a partitioned heterogeneous network in order to provide at least 1-connectivity. A simulation based study of the two schemes shows that both schemes can correctly add links to provide connectivity, but SMS is easier to implement with the drawback of a higher cost.

\section{REFERENCES}

[1] I. F. Akyildiz and X. Wang, "A survey on wireless mesh networks", IEEE Communications Magazine, Vol. 43, No. 9, pp. S23-S30, Sept., 2005.

[2] H. Moustafa and Y. Zhang, Vehicular networks, techniques, standards, and applications, CRC Press, 2009.

[3] I. Chlamtac, M. Conti, and J. J. Liu, "Mobile ad hoc networking: imperatives and challenges," Ad Hoc Networks, vol. 1, no. 1, pp. 13-64, July, 2003.

[4] P. Gupta and P.R. Kumar, "Critical Power for Asymptotic Connectivity in Wireless Networks," Stochastic Analysis, Control, Optimization and Applications, Boston: Birkhauser, pp. 547-566, 1998.

[5] P. Santi and D.M. Blough, "The Critical Transmitting Range of Connectivity in Sparse Wireless Ad Hoc Networks," IEEE Transactions on Mobile Computing, Vol 2. No. 1, January-March 2003

[6] F. Xue and P.R. Kummar, "The Number of Neighbors Needed for Connectivity of Wireless Networks," Wireless Networks 10, Kluwer Academic, Netherlands, 2004, pp. 169-181

[7] R. Hekmat and P. V. Mieghem, "Study of connectivity in wireless ad-hoc networks with an improved radio model," In Workshop on Wireless Optimization, WiOpt, 2004.

[8] T.-H. Kim, D. Tipper and P. Krishnamurthy, "Connectivity and Critical Point Behavior in Mobile Ad Hoc and Sensor Networks," Proceedings of IEEE ISCC'09, July, 2009.

[9] C. Bettstetter, "On the minimum node degree and connectivity of a wireless multihop network," Proc. 3rd ACM International Symposium on Mobile Ad Hoc Networking and Computing (MobiHoc), Lausanne, Switzerland, pp. 80-91, June 9-11 2002.

[10] Q. Ling and Z. Tian, "Minimum Node Degree and k-Connectivity of a Wireless Multihop Network in Bounded Area," Proceedings of IEEE Globecom, 2007.

[11] H. Zhang and J. C. Hou, "On the critical total power for asymptotic k-connectivity in wireless networks," IEEE/ACM Transactions on Networking, April, 2008.

[12] D. Kotz, C. Newport, R.S. Gray, J. Liu, Y. Yuan, and C. Elliott, "Experimental Evaluation of Wireless Simulation Assumptions", Proceedings of the $7^{\text {th }}$ ACM International Workshop on Modeling Analysis and Simulation of Wireless and Mobile Systems (MSWiM 2004), Venice, Italy, Oct., 2004.

[13] F. Kuhn and A. Zollinger, "Ad-hoc networks beyond unit disk graphs," in Proc. of the ACM Workshop on Foundations of mobile computing, 2003.

[14] C. Bettstetter and C. Hartmann, "Connectivity of Wireless Multihop Networks in a Shadow Fading Environment," Wireless Networks 11, Springer Science, 2005.

[15] C. Godsil and G. Royle, Algebraic Graph Theory, Springer-Verlag, 2001.

[16] C. Savarese, J. M. Rabaey, and J. Beutel, "Location in Distributed Ad-Hoc Wireless Sensor Networks," Proc. IEEE Int'l Conf. Acoustics, Speech, and Sgnal Processing, Vol. 4, pp. 2037-2040, May 2001.

[17] T. He, C. Huang, B. M. Blum, J. A. Stankovic, and T. Abdelzaher, "Range-Free Localization Schemes for Large Scale Sensor Networks," Proc Ninth ACM Int'l Conf. MOBICOM, pp. 81-95, Sept. 2003. 